\title{
Mitteilungen der DGU
}

Urologe $2021 \cdot 60: 657-665$

https://doi.org/10.1007/s00120-021-01538-0

(c) Springer Medizin Verlag GmbH, ein Teil von Springer Nature 2021

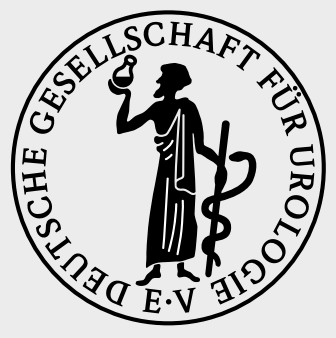

Herausgegeben vom Vorstand der

Deutschen Gesellschaft für Urologie

Schriftführer

Prof. Dr. med. C. Wülfing, Hamburg

Schriftleitung

Rechtsanwalt F. Petersilie, LL.M. (V.i.S. d.P.)

Geschäftsstelle der Deutschen Gesellschaft für Urologie e.V.

Uerdinger Str. 64

40474 Düsseldorf

\section{Inhalt}

657

658

661

688

689

693

Europaflagge über 73. Urologen-Kongress

Wie gut kennen Sie die Geschichte Ihres Faches?

Wissenschaftliche Preise der DGU

Fortbildungstermine der DGU

Akademie-zertifzierte Veranstaltungen

Kongressankündigungen

\section{PRESSEINFORMATION}

\section{Europaflagge über 73. Urologen- Kongress}

\section{DGU-Präsident Stenzl forciert internationale Zusammenarbeit in der Urologie}

Berlin/Düsseldorf. Während die einen den Brexit vollziehen, steht mit Prof. Dr. Dr. h.c. Arnulf Stenzl ein überzeugter Europäer an der Spitze der deutschen Urologie. Der amtierende Präsident der Deutschen Gesellschaft für Urologie e. V. (DGU) hat sich seit Jahren der internationalen Zusammenarbeit in der Urologie verschrieben, ist, neben seinem Engagement in der deutschen Fachgesellschaft, Adjunct Secretary Science der European Association of Urology (EAU) und Mitglied des Leitungsgremiums der European Cancer Organisation (ECO). In diesem Geiste setzt der ärztliche Direktor der Klinik für Urologie, Tübingen, auf dem 73. DGU-Kongress, der unter seiner Leitung vom 15. bis 18. September 2021 in Stuttgart tagt, auch einen europäischen Schwerpunkt.

„Die Vernetzung der Urologie in Deutschland mit den Nachbarländern bietet Chancen in Wissenschaft, Ausbildung und Patientenversorgung, die wir besser als bisher nutzen können. Dazu gehört auch, dass wir uns vermehrt um Dinge wie Gesetzgebung und finanzielle Unterstützung, die in den Gremien der EU in Brüssel entschieden werden, kümmern müssen, um die europäische und damit die deutsche Urologie zu stärken“, sagt Prof. Stenzl. Beispiele hat er parat: „Während der gemeinsame Bundesausschuss (G-BA) trotz aller Interventionen der DGU, hochkarätiger Institutionen und zahlreichen nationalen wie internationalen Fachgesellschaften sowie dem Bundesverband Prostatakrebs Selbsthilfe (BPS) Ende 2020 gegen den PSA-Test als Teil der gesetzlichen Prostatakrebsfrüherkennung in Deutschland entschieden hat, kommen aus Europa positive Signale. So sieht die EU-Kom- mission in ihrem jüngst veröffentlichten Europäischen Krebsplan eine Aktualisierung ihrer Empfehlungen zur Krebsfrüherkennung vor und wird Prostatakrebs möglicherweise in ihre Früherkennungsprogramme aufnehmen."

Auch bei Innovationen ist europäische Zusammenarbeit angezeigt, so der DGU-Präsident: „Nach wie vor dauert die Zulassung eines Medikamentes noch relativ lange. In meiner Eigenschaft als Mitglied des Leitungsgremiums der ECO, habe ich das auch schon bei der Gesundheitskommissarin Stella Kyriakides bei der europäischen Kommission angemahnt. Hier tut sich aber einiges: Im Bereich des Prostatakarzinoms in fortgeschrittenen frühen Stadien, wo ja erfreulicherweise eine längere Lebenserwartung zu sehen ist, wird von den Behörden zum Teil nicht mehr auf ein Gesamtüberleben gedrängt, sondern man akzeptiert in einigen Fällen für eine Zulassung auch das progressionsfreie Überleben (PFS). Damit kann die Zeit bis zu einer Zulassung aufgrund dieser Daten doch deutlich verkürzt werden."

Leitlinienkooperation mit der EAU sparen, nach Stenzls Worten, Ressourcen und nehmen bereits konkrete Form an: „Das DGU-Vorstands-Ressort für Leitlinien und Qualitätssicherung arbeitet schon eng an gemeinsamen Leitlinienerstellungen mit dem Guidelines Office der EAU zusammen."

Das Bild der deutschen Urologie aus europäischer Sicht wird übrigens der Generalsekretär der Europäischen Gesellschaft für Urologie, Prof. Christopher Chapple, in seinem Eröffnungsvortrag auf dem 73. DGU-Kongress im Herbst in Stuttgart zeichnen.

Dass die nationale und internationale urologische Community auf der weltweit drittgrößten Tagung des Fachgebietes in diesem Jahr wieder in physischer Anwesenheit zusammenkommt, steht für den Kongress-Präsidenten fest: „Wir setzen beim 73. DGU- Kongress in Stuttgart ganz klar auf eine Präsenzveranstaltung. Dies stützt sich zum einen auf der Tatsache, dass es bereits jetzt in den großen Räumlichkeiten des Internationalen Congresscenters Stuttgart ein ausgeklügeltes Hygienekonzept gibt. Bis zum Sommer werden außerdem das gesamte medizinische Personal und weite Teile der Bevölkerung durchgeimpft sein. Und letztendlich findet der Kongress eine Woche vor der Bundestagswahl statt ... Es wird aber in Stuttgart ein neues, innovatives Konzept eines umfassenden Videostreamings geben, das wir, wie bei vielen anderen großen Kongressen zahlungspflichtig anbieten werden. Es wird Teilnehmende nicht davon abhalten, die Vorteile einer Präsenzveranstaltung zu nutzen, ermöglicht es aber, dass Besucher, die zum Beispiel berufsbedingt nicht an allen vier Tagen des Kongresses anwesend sein können, Teile des wissenschaftlichen Programms von daheim vor dem Computer verfolgen können“.

Unter dem Kongressmotto „eUrologie“ hat Prof. Stenzl neben Europa und der „Eurologie“ auch den digitalen Fortschritt im Blick. „Di- 
gitale Medizin, Tele-Health, künstliche Intelligenz - auch diese Themen werden entsprechend dem Motto des Kongresses einen breiten Raum einnehmen. Namhafte Redner bis hin zu Beratern der Bundesregierung werden Konzepte und Bedürfnisse der Urologie diskutieren“, sagt der DGU-Präsident, der zudem die €logie ins Visier nimmt. „Bezüglich der allgemeinen Kosteneinsparung würden wir gut daran tun, selbst Kosten dort zu reduzieren, wo es weder den Patienten noch uns Urologen weh tut, aber vor allem zu keinen Einschnitten bei der Qualität der urologischen Patientenversorgung führt.“ Der renommierte Uro-Onkologe mit Schwerpunkt rekonstruktive Urologie betont: „Gerade die onkologische Urologie muss heutzutage gemeinschaftlich und in Teamarbeit im multidisziplinären Tumorboard diskutiert und entschieden werden. Hier ist es wichtig, dass der urologische Vertreter auch in den nicht-chirurgischen und interventionellen Behandlungsmöglichkeiten und Behandlungssequenzen seiner onko-urologischen Patienten ausgebildet ist. Deutschland nimmt dabei eine Vorreiter- und Vorbildfunktion in der Tätigkeit und Ausbildung uro-onkologischer Kollegen ein. Wir sollten das bisher Erreichte aber nicht nur erhalten, sondern weiter ausbauen zum Wohle der Patienten, aber auch zur Einsparung von Ressourcen. Patienten müssen nicht im Rahmen ihrer Behandlung mehrfach zwischen Kollegen verschiedener Disziplinen hin und her wechseln."

Im Kongressprogramm ist die Uro-Onkologie auch 2021 stark vertreten: etwa mit mehreren Updates der onkologischen S3-Leitlinien für die Praxis und Erweiterungen in der Diagnostik, zum Beispiel bei Hodentumoren, der urinbasierten Diagnostik beim Blasen- und Prostatakarzinom sowie der Bildgebung.

Neue Erkenntnisse erwartet Prof. Stenzl u. a. auch bei Methoden der minimalinvasiven Therapie gutartiger Erkrankungen wie der BPH und der Inkontinenz, in der Kinderurologie und der Infektiologie. Aktuelle Highlights setzt er mit zahlreichen hochkarätigen Kursen unter anderem zum Beurteilen von MRT, zur genetischen Beratung, zu Hygieneerfordernissen und zum Impfwissen, das den Urologen als Impfarzt stärken soll.

Viralen Infektionen widmet der überzeugte Verfechter einer europäisch vernetzten Urologie in Zeiten der Coronavirus-Pandemie ein Plenum mit herausragenden Gastrednern. „Nobelpreisträger Professor Harald zur Hausen bringt uns neue Erkenntnisse in der Behandlung von Prostata-, Colon- und Mammakarzinom und Professor Peter Kremsner, Leiter der klinischen Studien für das Curevac-Vakzin, wird die Entstehung eines mRNA-basierten COVID-19-Impfstoffes aus eigener Erfahrung beleuchten“, sagt DGU-Präsident Stenzl, der mit einer organisatorischen Fahrplanänderung im traditionellen Kongressablauf überrascht und den 15. September 2021, an dem die 73. Jahrestagung der Fachgesellschaft ihre Türen im Internationalen Congresscenter Stuttgart (ICS) Fachbesuchern aus aller Welt öffnet, definitiv zu einem vollwertigen Kongresstag erklärt. „Dem Wunsch der Teilnehmerinnen und Teilnehmer vergangener Jahre entsprechend wird der Mittwoch ein vollwertiger Kongresstag, an dem am Nachmittag auch das Eröffnungsplenum stattfindet."

\section{Weitere Informationen}

DGU-Kongress-Pressestelle

Bettina-Cathrin Wahlers

Sabine Martina Glimm

Wettloop 36c

21149 Hamburg

Tel.: $040-79140560$

Mobil: $0170-4827287$

E-Mail: redaktion@bettina-wahlers.de

\section{Wie gut kennen Sie die Geschichte Ihres Faches?}

\author{
Eine Zeitreise durch das einzigartige Museum der \\ Urologie in Berlin
}

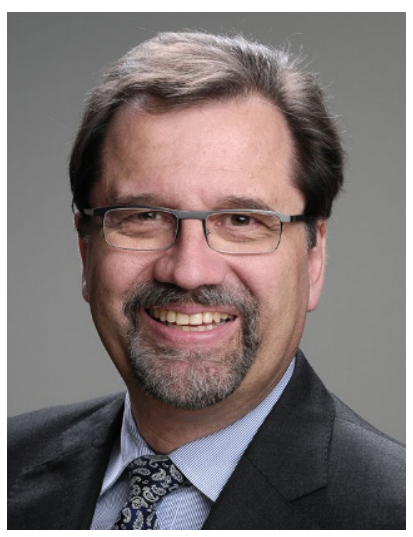
Große Instrumente zur Entfer- nung von Harnsteinen, historische Schriften oder ein 28.000 Jahre altes Phallus-Idol: Museum, Bi- bliothek und Archiv im Haus der Urologie in Berlin-Zehlendorf zei- gen seltene Schätze und Kuriosa aus der Geschichte und Kultur der Urologie. PD. Dr. Friedrich Moll, Kurator des Museums der Deut- schen Gesellschaft für Urologie e.V., gibt ungewöhnliche Einbli- cke rund um die wissenschaftliche Ausstellung.

1. Herr PD Dr. Moll, die Behandlung urologischer Erkrankungen zählt zu den ältesten ärztlichen Tätigkeiten: Seit wann werden Blasenleiden, Nierensteine und $\mathrm{Co}$. behandelt?

Die Dokumentation von Erkrankungen im Uro-Genitalbereich und deren Behandlung lässt sich weit zurückverfolgen, wenn auch manchmal eine retrospektive Diagnose anhand von Schriften oder Bildquellen schwierig ist. Fest steht, Harnsteine und auch die Beschneidung lassen sich bereits bei ägyptischen Mumien nachweisen. Die Verabreichung von potenzfördernden Mitteln reicht noch früher bis in die Zeit der Priester-Ärzte zurück.

\section{Der "goldene Strich" oder tödliche Potenzmittel aus der gemahle-} nen Spanischen Fliege sind legendär: Wie gefährlich waren solche Behandlungen?

In der Tat war die Behandlung eines Wasserbruches, einer Hydrozele, nur durch Punktion möglich, die jedoch entfernt vom Hoden gesetzt werden musste und damit schon eine gute Fingerfertigkeit, gerade in der voranästhetischen Zeit voraussetzte.

„Spanische Fliegen“, Canthariden der Gattung Lytta vesicatoria, waren bis zu Beginn des 20. Jahrhunderts gängige Aphrodisiaka, die in Apotheken angeboten wurden. Sie sind ein starkes Reiz- und Nervengift, wodurch es als Wehrsekret in der Tierwelt sehr effektiv ist. Auf der Haut und vor allem auf den Schleimhäuten des Menschen übt es eine starke Reizwirkung aus. Beim Menschen und bei anderen Wirbeltieren löst das enthaltende Gift die Bildung von Blasen und teilweise tiefen Hautwunden aus. Außerdem führt es zu Entzündungen und insbesondere zu einer starken Schädigung der Nieren. Letztere tritt vor allem bei Missbrauch, etwa bei übermäßiger Einnahme als Aphrodisiakum, auf. Gerade, da es sich um ein Naturprodukt handelt, war eine Dosierung immer schwierig. Heute kann die Urologie ihren Patienten deutlich sicherer zu dosierende Medikamente anbieten, deren Einführung vor über 20 Jahren auch schon zur Geschichte gerinnt.

\section{Und bis wann wurde der sogenannte Impotenz-Gürtel eingesetzt?}

Die „Impotenz-Gürtel“ oder „Heidelberg Belts“ gehören zu den besonders in den USA stark nachgefragten „Universalheilmitteln“ bei sexueller Neurasthenie zu Beginn des 20. Jahrhunderts. Die Neura- 
sthenie war eine „Modediagnose“ am Ende des 19. und zu Beginn des 20. Jahrhunderts, nachdem man die Nervenleitung als elektrische Impulse beschrieben hatte und man sich durch Elektrizität in diesem Organbereich eine besondere Therapiemöglichkeit erhoffte. Da dieser Erkrankungskomplex auch mit männlichem „Unvermögen“ assoziiert wurde, erhoffte man sich hier eine moderne Therapieform. Die Diagnose „sexuelle Neurasthenie“ bot darüber hinaus zu Zeiten einer doch prüden Gesellschaft die Möglichkeit, über Sexualität, Liebe und Ehe überhaupt zu sprechen und fachlichen Rat einzuholen. Die mannigfachen von Laien, Medizinern und Urologen verfassten „Aufklärungsschriften" veranschaulichen die riesige Breite dieses Gebietes bis in die 1920er Jahre.

4. Neben einem Schatz aus tausenden Publikationen, zahlreichen Original-Lithografien und Dokumenten zeigt die Sammlung des Museums rund 1500 historische Instrumente, lässt in eine urologische Praxis aus der Mitte des letzten Jahrhunderts blicken und liefert zweifellos ein starkes Plädoyer für die urologische Gegenwart: Welches sind die wichtigsten Exponate aus medizin-historischer Sicht?

Eine einfache und zugleich schwierige Frage für den Museumsfachmann, da ja alle Objekte eine eigene Geschichte erzählen, sei es ein Steinschnitt-Besteck, das ein bayrischer Urologen seinerzeit wegen seiner Verdienste und langjährigen Tätigkeit vom Bayrischen Prinzregenten erhielt und das die Bedeutung und das Ansehen des sich gerade entwickelnden „neuen“ Arztspezialberufes unterstreicht. Aus Sicht der medizinischen Fachgesellschaft sind es wenige, optisch spektakuläre Schriftstücke, die die Gründung im Jahre 1907 dem „Preußischen Minister der Geistlichen-Kultus und Medizinalangelegenheiten“ anzeigen. Für den Besucher besonders beeindruckend ist sicherlich das große Set zur „blinden“ - modern ausgedrückt „minimal invasiven Behandlung von Blasensteinen“, das von einem zu seiner Zeit sehr bekannten Pariser Instrumentenmacher um 1830 angefertigt wurde. Für den Wissenschaftshistoriker sind es u.a. unsere exquisiten Stiche, die einen Querschnitt aus mehreren Jahrhunderten der Fachentwicklung dokumentieren und die Sicht auf die Erkrankung und den Patienten visualisieren. Im Archiv sind es die eher unscheinbaren Aktenkonvolute, die urologischen Alltag auf vielfältigen Ebenen beschreiben.

Bei dieser Frage können bei der Planung von Ausstellungen auch einmal die Ansichten von Kurator und Kustos auseinandergehen.
Während der Kurator eher die fachkonstituierenden Artefakte fokussiert, sind unter kustodialen Gesichtspunkten häufig die kleineren, unscheinbareren Objekte im Mittelpunkt, da diese oft starke, spannende Geschichte von Urologen als Sammlern oder Patientengeschichten multidimensional erzählen.

\section{Welches sind die skurrilsten Ausstellungsstücke?}

Was uns heute als sonderbar oder verschroben erscheint, war häufig unter anderen wissenschaftlichen und gesellschaftlichen und kulturellen Vorzeichen gängig, häufig und populär. Wir sollten daher vorsichtig sein, mit unserem heutigen Blick historische Diagnosen und Behandlungen zu interpretieren, denn das hieße immer einen anderen Betrachtungshorizont einzunehmen.

Betrachtet man Artefakte unter dem Aspekt der Besonderheit oder Einzigartigkeit sind es nach meiner Überzeugung die römischen Spintriae/tesserae, die zurzeit vor allem als Spiel- und als Bordellmarken gedeutet werden und unseren modernen Jetons entsprechen dürften. Kontinuitäten seit mehr als 2000 Jahren!

\section{Wie reagieren die Besucher auf die historischen Gerätschaften?}

Vielen Dank für diese Frage, denn hier müssen eindeutig zwei Besuchergruppen unterschieden werden. Fachbesucher kennen vom Prinzip die meisten Instrumente von ihrer aktuellen Tätigkeit und sind eher verwundert, dass an vielen Prinzipien wenig geändert wurde. Wollen sie dann ein Instrument ausprobieren, was möglich ist, sind sie bei Zystoskopen verwundert, dass das Bild so lichtschwach ist und auf dem Kopf und spiegelverkehrt erscheint. Sie müssen das „Sehen“ neu lernen. Auch jahrzehntelang trainierte Urologen haben dann wieder eine Lernkurve wie die Studenten in unserem Düsseldorfer Seminar „Op trifft Museum“, das Medizinstudenten ermöglicht, neben dem Erlernen wissenschaftlicher Techniken mit alten und neuen Zystoskopen am selbst gebauten Phantom zu untersuchen, was das klinischen Praktikum mit Tastkurs nicht bietet.

Interessierte Besucher ohne urologischen Hintergrund sind häufig aufgrund der Instrumentenform beunruhigt und antizipieren eine schmerzhafte Untersuchung, die es heute nicht gibt. Hier dient das Museum als Mittler zur aktuellen Urologie, der ansprechend „verpackte" Fachinformationen zu Themen liefert, die viele auch heute nicht öffentlich fragen würden. Reaktionen liegen somit immer im Auge des Betrachters.



7. Haben Harnsteine tatsächlich den Lauf der Geschichte beeinflusst und Napoleons Erkrankungen Schlachten entschieden?

Dies kann man eindeutig mit Ja beantworten, wobei dieser Aspekt gerade von französischen Historikern gerne bei Napoleon III bemüht wird. Napoleon III litt an einem Blasenstein und hier ist eine retrospektive Diagnose ausnahmsweise möglich, da die Steine noch heute in London archiviert sind. Der Herrscher verlor eine wichtige Schlacht, da der Einsatzbefehl zu spät gegeben wurde. Dies ermöglichte jeweils der preußischen Armee einen gewissen Vorsprung in der Schlachtaufstellung, was franzö- 


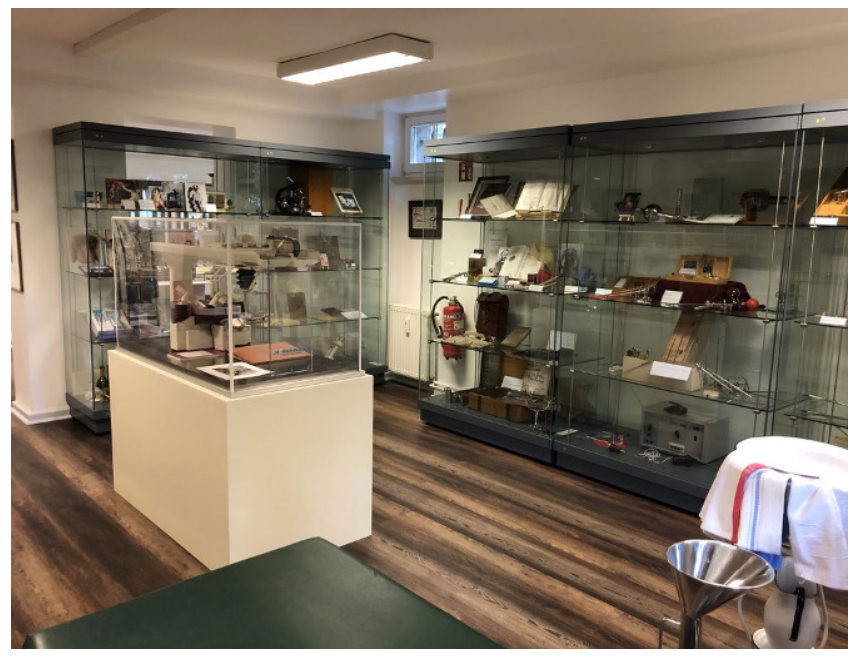

sische Historiker den Fieberschüben des Herrschers bei der Schlacht zuschrieben. Wie man aber weiß, führt ein Detail allein sicherlich nicht zu Sieg oder Niederlage bei einer Schlacht. Das Narrativ wird aber bis heute gerne in der national geprägten Literatur genutzt. Man sollte die Geschichte eher fachpolitisch erzählen. Obwohl Frankreich zu den frühen führenden Ländern mit einer sich spezialisierenden Urologie gehörte, wurde der Kaiser in diesem Bereich eher unterversorgt, was sicherlich suboptimal war und nicht den fachlichen Möglichkeiten zu seiner Zeit entsprach. Paris war im 19. Jahrhundert ein Mekka zur Weiterbildung in der Spezialität Urologie. Der Franzose Napoleon III wurde im Exil in England von einem Briten operiert. Nationale Gegensätze prägten die Politik im 19. Jahrhundert wesentlich.

\section{Gibt es weitere berühmte urologische Patienten in der Vergangen- heit?}

Dies ist immer wieder ein Thema von Vorträgen, wobei, wie schon erwähnt, häufig eine retrospektive Diagnose schwierig und allenfalls bei Harnsteinpatienten wie Martin Luther oder auch Ava Gardner und u. a. Jeff Bezos gesichert ist. Im Internet findet sich eine Wikipedia Liste „List of people with kidney stones“ aus allen Gesellschaftsbereichen. In früheren Zeiten konnten sich nur begüterte Menschen Fleisch und Wildbret leisten, deren Erkrankungen bis zu den ausgeschiedenen Nierensteinen dann auch gut dokumentiert wurden. Das erkrankte Messnerkind Zuttel, dessen Blasenstein von Lorenz Heister behandelt wurde und worüber eine Korrespondenz mit dem Vater existiert, aus der wir einen guten Einblick in das Patientenschicksal erhalten haben, findet sich in solchen Listen nicht. Auch die Anamnese von Politikern mit prostatischen Beschwerden, die einen harten Wahlkampf nur mit Katheter durchstehen konnten und sich währenddessen nicht operieren lassen konnten oder wollten, faszinieren zwar ein allgemeines Publikum, sind aber eher ein Beispiel, wie eine Behandlung eher suboptimal abläuft. Werden Politiker dann initial an der falschen Seite bei renalen Erkrankungen operiert oder leiden gar an Prostatakarzinomen besitzen diese Fakten zwar einen gewissen Unterhaltungswert für die Boulevardpresse, sollten aber doch eher unter die ärztliche Schweigepflicht fallen.

\section{Einst Harnbeschauer und Steinschneider, heute Fachärzte für Urolo- gie, die Kl und OP-Roboter nutzen: Welches sind die wichtigsten medizi- nischen Fortschritte und Errungenschaften auf dieser Zeitreise? \\ Es fällt mir schwer, die Entwicklung als Fortschrittsgeschichte zu beschreiben, wie es „Old School“-Medizinhistoriker und manche Uro-}

logen in Handbuchbeiträgen häufig bis in die Mitte des 20. Jahrhunderts taten. Diese Sichtweise ist heute verlassen und gilt als unwissenschaftlich.

Urologen und Proto-Urologen haben sich früh auf ausgesuchte, mit dem Harntrakt verbundene Erkrankungen spezialisiert und damit die Brücke von der handwerklichen zur naturwissenschaftlichen Ausbildung schlagen können. Bereits die alten Medizinalordnungen deutscher Fürstentümer erwähnten und regelten Steinschneider bzw. "Lithotomisten“ in ihrer Berufsausübung und auch Liquidation separat. Dr. Eisenbarth oder Frère Jacques sind gerade aufgrund ihrer für ihren Zeitraum niedrigen Operationsletalität in der allgemeinen Erinnerungskultur erhalten geblieben, auch wenn sie im 19. Jahrhundert teils als ungebildet und roh beschrieben wurden. Urologen haben ein minimal invasives Vorgehen schon sehr früh im 19. Jahrhundert favorisiert, da sie hiermit deutlich messbare und statistisch auswertbare Überlebensvorteile bei ihren Eingriffen besaßen. Durch die Beschränkung auf ein Organsystem konnten Urologen, wie schon ausgeführt, viel früher als andere medizinische Fachgebiete ein funktionelles Denken in ihrem Wissenschaftsbereich einführen, was ihnen ein besseres Verständnis von Zusammenhängen von Körperfunktionen ermöglichte. Aufgrund ihrer Interdisziplinarität, das Fachgebiet speiste sich aus offen-operativ am Harntrakt Interessierten, Endoskopikern, Sexualmedizinern, Dermatologen, an den allgemeinen Nierenerkrankungen Interessierten und dem weiblichen Harntrakt Interessierten, konnte sich eine Fachdisziplin entwickeln, die auch heute zu besonders vielen Bereichen der medizinischen Wissenschaften anschlussfähig ist und neue Forschungsfragen generieren kann. Somit wurde das, was Sie als Fortschritt bezeichnen in jeder Generation neu generiert und fortgeschrieben.

10. Die Anfänge der heutigen Sammlung lagen in den 1960er Jahren in Berlin, es folgte Düren, dann der Museumsstandort in Düsseldorf und schließlich 2019 Berlin: „Museum, Bibliothek und Archiv zur Geschichte der Urologie" haben ihre eigene Geschichte und in der Deutschen Gesellschaft für Urologie e. V. eine wichtige wissenschaftliche Funktion. Welche Aufgaben sind das?

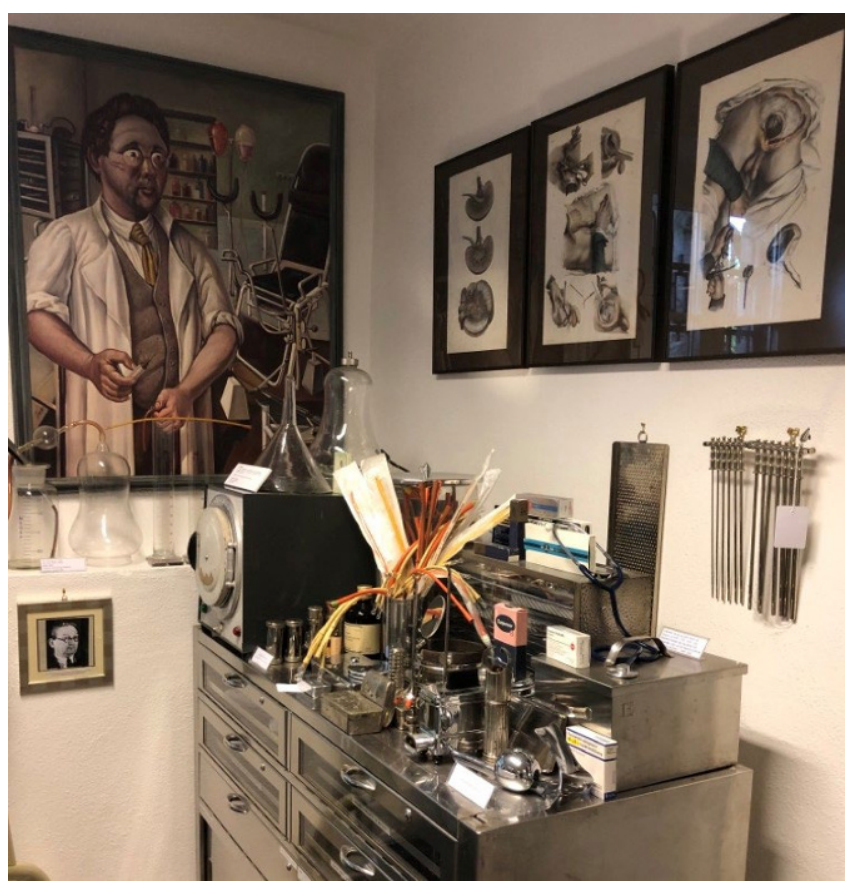


Sammeln, Bewahren, Forschen sind die Hauptaufgaben jedes Museums und natürlich auch unserer Einrichtung. Die Unterhaltung von Museum, Bibliothek und Archiv sind ein Alleinstellungsmerkmal der wissenschaftlichen Fachgesellschaft, nur wenige wissenschaftliche Fachgesellschaften unterhalten ein Museum, eine Bibliothek und ein Archiv in diesem Rahmen. Dies kann als ein wesentliches Exzellenzkriterium gewertet werden. Die Deutsche Gesellschaft für Urologie e. V. trägt dem mit den Positionen Kurator und Kustos sowie Archivar besondere Rechnung, die eine wissenschaftliche und technische Betreuung dieser einmaligen wissenschaftshistorischen Sammlung ermöglichen und fortschreiben.

11. Auch mit der Präsentation thematischer historischer Ausstellungen auf den Jahrestagungen der DGU hält die Fachgesellschaft die Geschichte der Urologie lebendig: Was ist für den 73. Kongress im September 2021 in Stuttgart geplant?

Nachdem die wissenschaftshistorische Ausstellung 2020 dem Publikum leider „nur“ virtuell präsentiert werden konnte, hoffen wir 2021 in Stuttgart wieder „live“ dabei sein zu können und entsprechend dem Kongress-Motto eUrologie und dem Kongress-Standort Stuttgart unseren Besuchern, Freunden und Förderern eine Kollektion von Objekten zur Entwicklung der süddeutschen Urologie sowie zur Wissenskommunikation zu präsentieren. Weiterhin wollen wir auf unsere Spender blicken, die uns Material in vielfältiger Form von Akten bis Filmen, von Instrumenten bis Ephemera dankenswerter Weise überlassen haben. Denn ohne unsere Spender wäre dies alles nicht möglich geworden.

12. Wer besucht das Berliner Urologie-Museum? Ärzte, Studenten, Schülergruppen, das breite Publikum? Gibt es spezielle Kooperationen - auch die Urologie braucht schließlich Nachwuchskräfte?

Wir haben Besucher aus allen angesprochenen Kreisen. Es gibt Kooperationen zu Ausbildungseinrichtungen für den technischen Nachwuchs bei der Charité. In unserem Studentenkurs in Düsseldorf, am Ort der Düsseldorfer Geschäftsstelle und des Depots an der Uerdinger Strasse, sind wir somit am Puls des ärztlichen Nachwuchses.

13. Wie groß ist das Interesse am heutigen Museums-Standort in Berlin, und was erwartet die Besucher? Infos über den Knopf im Ohr, oder eine persönliche Führung?

Wegen des coronabedingten Lockdowns können leider schon länger keine Führungen mehr stattfinden. Es ist bei der derzeitigen Dynamik sowohl seitens des Infektionsgeschehens als auch der verhängten Maßnahmen auch nicht absehbar, wann sich dieses verlässlich wieder ändert. Deshalb müssen wir Interessierte aktuell vertrösten und können sie lediglich auf einer Warteliste registrieren. Dafür bitten wir um Verständnis. Anmeldungen sind unter der Telefonnummer 030/88 70 833-0 oder per E-Mail an info@dgu.de oder an keyn@dgu.de möglich. Wenn sich die Situation geändert hat, werden sowohl Kustos Jörg-Michael Moll-Keyn als auch ich als Kurator die persönlichen Museumsführungen wieder aufnehmen und die Geschichten hinter den Exponaten wie bisher besucherorientiert erzählen. Mittelfristig ist zudem die Anschaffung eines Audioguides geplant, um das Angebot für unsere Besucher weiter zu verbessern.

\section{Interview: DGU-Pressestelle}

\section{Wissenschaftliche Preise der DGU}

\section{Wissenschaftliche Preise}

Für alle Bewerbungen zu den wissenschaftlichen Preisen gilt:

- Ende der Einreichungsfrist: 1. Juni

- Bewerbungen bitte an: preise@dgu.de

\section{Maximilian Nitze-Preis}

Der Maximilian Nitze-Preis erinnert an den in Dresden, Wien und Berlin tätigen Erfinder des Zystoskops und frühen Inhaber einer Professur für Urologie in Deutschland, Maximilian Nitze (1848-1906). Der Preis ist die höchste wissenschaftliche Auszeichnung der DGU und wird für herausragende wissenschaftliche Leistungen in der experimentellen oder klinischen Urologie vergeben.

Eingereicht werden können Habilitationsschriften und Originalarbeiten. Für Originalarbeiten gilt, dass diese nach dem vorletzten Kongress der Deutschen Gesellschaft für Urologie veröffentlicht oder zur Publikation akzeptiert sein müssen. Die Annahme durch eine Fachzeitschrift mit peer-review muss nachgewiesen werden. Der Preis ist mit $5000 €$ dotiert. Über die Preisvergabe entscheidet eine Jury.

\section{Wolfgang Knipper-Preis}

In Gedenken an den Hamburger Urologen Wolfgang Knipper (19202005) wird dieser Preis auf der Jahrestagung der Deutschen Gesellschaft für Urologie e. V. an Vertreter der Pflege- und Assistenzberufe vergeben, die sich in besonderer Weise durch persönliches, soziales oder wissenschaftliches Engagement in der Fort- und Weiterbildung ausgezeichnet haben.

Der von der Firma Fresenius Kabi unterstützte Preis ist mit $3000 €$ dotiert.

Über die Vergabe des Preises entscheidet eine Jury.

\section{Dora Teleky-Preis}

Der Dora Teleky-Preis wird von der Deutschen Gesellschaft für Urologie e. V. an besonders qualifizierte und wissenschaftlich renommierte Urologinnen verliehen.

Der Preis erinnert an die jüdische Wiener Urologin Dora BrückeTeleky (1879-1963), die 1911 als erste Frau Mitglied der Deutschen Gesellschaft für Urologie wurde und sich besonders mit uro-gynäkologischen Fragen beschäftigte. Sie führte eine gynäko-urologische Praxis in Wien, war als erste Schulärztin für gewerbliche MädchenFortbildungsschulen tätig und ab 1919 Leiterin der Schwangerenfürsorgestelle. Dora Brücke-Teleky gründete 1919 die Organisation „Ärztinnen Wiens" und engagierte sich als korrespondierende Sekretärin des „Internationalen Ärztinnenverbandes“. Im August 1939 wurde sie als jüdisch klassifiziert und gezwungen zu emigrieren.

Zur Bewerbung sind Fachärztinnen für Urologie mit herausragender Forschungsleistung aus Klinik oder Praxis aufgefordert. Gewürdigt wird die bisherige Leistung. Gleichzeitig soll die Auszeichnung die weitere wissenschaftlich-klinische Entwicklung der Preisträgerin fördern. Bewerben können sich sowohl Urologinnen, die bereits habilitiert sind als auch solche, die ihre Habilitation unmittelbar anstreben.

Der von der Firma Eisai unterstützte Preis ist mit $5000 €$ dotiert

Über die Vergabe entscheidet eine Jury.

\section{Alexander von Lichtenberg-Preis}

Der Alexander von Lichtenberg-Preis wird zur Erinnerung an einen der Pioniere der deutschen Urologie und Mitentwickler der Ausscheidungs-Urographie Alexander von Lichtenberg (1880-1949) verliehen. 
Er wird für besondere Leistungen im Bereich der ambulanten Urologie, vorrangig im niedergelassenen Bereich, vergeben.

Der von der Firma Takeda Pharma gestiftete Preis ist mit $5000 €$ dotiert. Über die Preisvergabe entscheidet eine Jury. Die Bewerbungsunterlagen stehen ab Frühjahr des lfd. Kalenderjahres unter www.alexander-von-lichtenberg-preis.de zum Download bereit.

\section{Ernst-Fürstenheim-Förderung ambulanter Forschungsprojekte}

In Erinnerung an Ernst Fürstenheim (1836-1904) - einem bedeutenden deutschen Urologen und Gründer der ersten Urologischen Praxis in Berlin - wird die Förderung für besondere wissenschaftliche Vorhaben auf dem Gebiet der ambulanten Patientenversorgung in der Urologie gewährt.

Die Ausschreibung richtet sich vorrangig an niedergelassene Urologinnen und Urologen sowie deren Mitarbeiterinnen und Mitarbeiter.

Ziel der Deutschen Gesellschaft für Urologie e. V. ist es, zukunftsweisende Modellvorhaben, Forschungsprojekte und wissenschaftliche Untersuchungen mit Schwerpunkt auf ambulante Behandlungs- und Versorgungsformen im Fachbereich Urologie in Deutschland zu fördern. Im Ergebnis sollen die Effizienz und Qualität der ambulanten Versorgung gestärkt und die Einführung von Innovationen unterstützt werden.

Die Ausschreibung ist an Einzelne oder eine Gruppe von Urologinnen und Urologen adressiert, die Mitglied der DGU sind und sich mit einem wissenschaftlichen Projekt bewerben, das als Präsentation auf der aktuellen, spätestens nächsten Jahrestagung der Deutschen Gesellschaft für Urologie vorgestellt wird.

Kriterien sind insbesondere die wissenschaftliche Originalität der Fragestellung, eine sehr gute methodische Qualität sowie eine hohe Relevanz für die Umsetzung in die ambulante Patientenversorgung.

Es sollte für das Forschungsvorhaben, sofern erforderlich, bereits ein positives Ethikvotum vorliegen.

Die Förderung ist mit insgesamt bis zu $20.000 €$ dotiert.

Über die Förderungsvergabe entscheidet eine Jury.

\section{Winfried Vahlensieck-Preis}

In Gedenken an den Bonner Urologen Professor Winfried Vahlensieck wird dieser Preis an Wissenschaftlerinnen und Wissenschaftler verliehen, die sich auf dem Gebiet der Erforschung des benignen Prostatasyndroms (BPS), deren Diagnose oder Therapie wissenschaftlich ausgezeichnet haben.

Der Preis ist mit $2000 €$ dotiert. Der von der Firma Omega Pharma unterstützte Preis ist mit $2000 €$ dotiert.

Über die Vergabe entscheidet eine Jury. 
Deutsche Gesellschaft für Urologie e.V.

Uerdinger Straße 64

40474 Düsseldorf

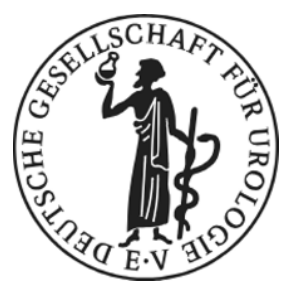

DEUTSCHE GESELLSCHAFT FÜR UROLOGIE E.V. AUFNAHMEANTRAG - Mitgliedschaften in der Deutschen Gesellschaft für Urologie e.V.

Ordentliches Mitglied kann jede Ärztin/jeder Arzt für Urologie (Fachärztin/Facharzt) werden. Assoziierte Mitglieder können Wissenschaftler(innen) werden, die keine Urologinnen/Urologen sind, aber Interesse für die Urologie zeigen. Die Deutsche Gesellschaft für Urologie e. V. bietet allen in der Weiterbildung befindlichen Kolleginnen und Kollegen eine Juniormitgliedschaft bis zu 5 Jahren an, die mit Abschluss der Weiterbildung in eine Vollmitgliedschaft übergeht. Die Aufnahme bedarf der schriftlichen Befürwortung durch zwei Mitglieder der Gesellschaft. Jedes Mitglied zahlt eine einmalige Aufnahmegebühr von EUR 25,-sowie jährlich den Mitgliedsbeitrag, dessen Höhe von der Mitgliederversammlung festgelegt wurde:

Ordentliche Mitglieder: EUR 160,-| Juniormitglieder: EUR 50,-| Assoziierte Mitglieder: EUR 160,- | Ausserordentliche Mitglieder: EUR 25,00

HIERMIT beantrage ich die

JUNIORENMITGLIEDSCHAFT (Noch in der Weiterbildung: Bitte Beginn und Ende der Weiterbildungszeit eintragen!)

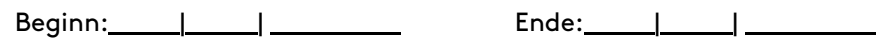

ASSOZIIERTE MITGLIEDSCHAFT (Kein Urologe, aber Interesse für die Urologie)

VOLLMITGLIEDSCHAFT (Facharzt)

AUSSERORDENTLICHE MITGLIEDSCHAFT (Pflege- und Assistenzberufe - Bei Antragsstellung sind keine Bürgen notwendig)

Titel: $\square$ ohne $\square$ Dr. $\square$ Priv.-Doz. $\square$ Prof. $\quad$ Name, Vorname

Geburtsdatum:

$\square$ Herrn $\square$ Frau

\begin{tabular}{|l|l|}
\hline Anschrift Klinik/Praxis/Dienststelle/privat: \\
\\
\hline Telefon/Fax: \\
\hline E-Mail:
\end{tabular}

Dürfen die oben genannten Adressdaten im Mitgliederverzeichnis erscheinen? $\square$ Ja $\square$ Nein Möchten Sie Mitteilungen der DGU an Ihre Emailadresse erhalten? Zusatzweiterbildungen:

2 Datum/Unterschrift des/der Antragsteller/in

MIT MEINER UNTERSCHRIFT BESTÄTIGE ICH DIE HINWEISE ZUR DATENERFASSUNG- UND VERARBEITUNG DER MITGLIEDERDATEN GELESEN UND VERSTANDEN ZU HABEN! Quelle: https://www.urologenportal.de/antrag

Hiermit befürworten wir die Aufnahme des vorgenannten Antragstellers in die Deutsche Gesellschaft für Urologie e.V. (Bitte leserlich in Druckbuchstaben ausfüllen) - Aufgeführte Bürgen müssen Mitglieder der Gesellschaft sein!
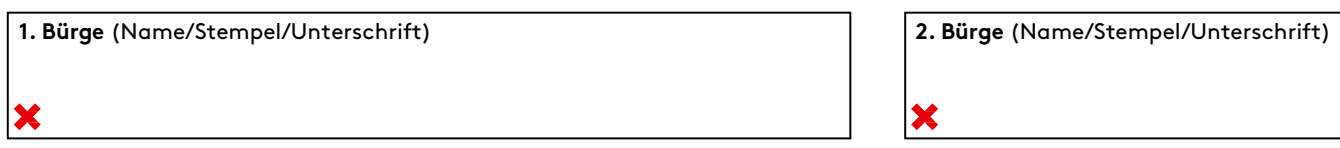

Erteilung eines SEPA-Lastschriftmandats, Gläubiger-Identifikationsnummer: DE43ZZZ00000553162. Ich ermächtige die Deutsche Gesellschaft für Urologie e.V., Zahlungen von meinem Konto mittels Lastschrift einzuziehen. Zugleich weise ich mein Kreditinstitut an, die von der Deutschen Gesellschaft für Urologie e.V. auf mein Konto gezogenen Lastschriften einzulösen. Hinweis: Ich kann innerhalb von 8 Wochen, beginnend mit dem Belastungsdatum, die Erstattung des belastenden Betrages verlangen. Es gelten dabei die mit meinem Kreditinstitut vereinbarten Bedingungen. Der Jahresbeitrag wird jedes Jahr im Januar eingezogen.

Name, Vorname Kontoinhaber)

Anschrift

IBAN (finden Sie auf Ihrem Konto-Auszug) Bank
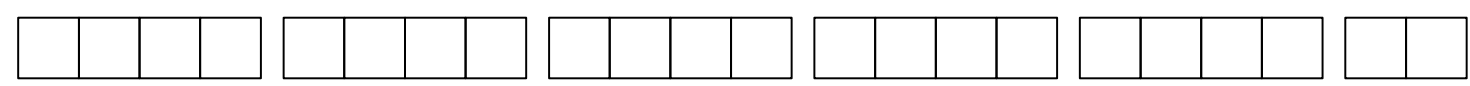


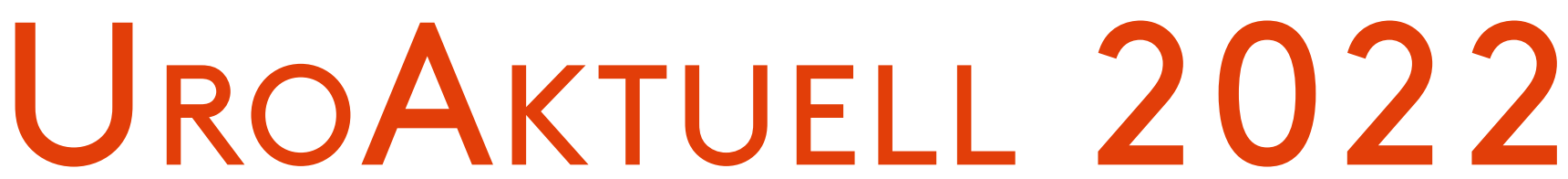
Aktuelles und Praxisrelevantes in der Urologie

$$
\text { 28. - 30. April } 2022 \text { I Berlin }
$$

\section{TAGUNGSHOTEL}

Holiday Inn Berlin - City West

Rohrdamm 80

13629 Berlin




